\title{
Mechanics for Vibratory Manipulation
}

\author{
Wesley H. Huang Matthew T. Mason \\ Robotics Institute \\ Carnegie Mellon University
}

\begin{abstract}
Vibratory manipulation is any mode of manipulation involving repeated impacts due to a striker which follows some periodic motion. In this paper, we study vibratory manipulation in the context of tapping planar objects which slide on a fixed support surface. We are interested in the behaviors an object exhibits under such excitation. There are two distinct types of tapping that can result: continuous tapping, in which the object is always in motion, and intermittent tapping, in which the object comes to rest between taps. We first examine vibratory manipulation in one dimension, adapting results from related work to find conditions for stable periodic motion. The general two dimensional case is closely related to our previous work in impulsive manipulation which examined the mechanics of a sliding rotating object. In fact, vibratory manipulation is an approximation to the limiting cases of impulsive manipulation. We develop the limiting cases for intermittent and continuous tapping for rotationally symmetric objects and conclude with some examples.
\end{abstract}

\section{Introduction}

Our previous work has focused on impulsive manipulation, i.e. manipulating objects through impulsive forces. In this paper, we explore a closely related topic: vibratory manipulation. Vibratory manipulation broadly refers to any manipulation process involving repeated impacts, generally at a high frequency, due to a striker which follows some regular or servoed periodic motion. In this paper, we consider vibratory manipulation in the context of manipulating planar parts that slide on a fixed support surface in between taps. Our interest is in stable periodic behaviors of an object that result from such excitation. There are many different scenarios for this sort vibratory manipulation that we can consider; these include:

- Open-loop control of a pusher with superimposed vibration. A pusher with a vibrating tip follows a predetermined trajectory while a striker tip executes some periodic motion. The actual object excitation is a high frequency, low amplitude sequence of collisions between the striker and the object.

- Feedback control of a pusher with superimposed vibration. Same as above except that the gross motion of the pusher is determined by some feedback control law.

- Feedback control of impulses. The object is sensed before every impact, and a control law determines an appropriate impulse to drive the object towards some goal configuration.

- Open-loop parts transfer. A sequence of impacts is planned to move an object from start to goal, assuming ideal behavior of the slider and perfect knowledge of all relevant mechanical parameters. The shape of the striker and the details of the striking motion can be designed so that small errors give rise to restoring impulses.

There are a number of possible applications for such manipulation relating to micropositioning and to parts feeding.

We find that there are two types of tapping that result under vibratory manipulation: intermittent tapping, where the object comes to rest in between taps, and continuous tapping, where the object is always tapped again before it comes to rest. Vibratory manipulation in one dimension that results in continous tapping is closely related to bouncing a ball and to robotic juggling. We review and adapt results from work in these areas to determine the conditions for which the object displays stable periodic motion.

Vibratory manipulation in two dimensions makes use of the same mechanics (of a sliding rotating planar object) as our previous work in impulsive manipulation. In fact, vibratory manipulation (which concerns object behavior from repeated impacts) approximates the limiting cases of impulsive manipulation (which concerns the mechanics of a single impact). In our efforts to formulate an understanding of the general case of vibratory manipulation, we have developed the limiting cases of impulsive manipulation for both intermittent and continuous tapping. We present detailed analysis of the mechanics of these limiting cases for rotationally symmetric objects. Finally, we apply the results of this analysis to determine the range of possible motions under these limiting cases for two different objects.

\section{Related Work}

Vibratory manipulation in which the support surface vibrates (and the object becomes airborne) is currently used 
in bowl feeders (see Boothroyd et al. [4] for descriptions and Boothroyd [3], Caine [6], and Christiansen et al. [7] for recent work on analysis and automated design for bowl feeders) and in the SONY APOS system (Hitakawa [10]). Böhringer $e t$ al. [2] oriented planar parts by taking advantage of the vibrational modes of the support surface.

The vibratory manipulation which we describe in this paper (involving a sliding phase of the object in between taps) is closely related to the problem of bouncing a ball on a vibrating table and to robotic juggling. The bouncing ball problem has been analyzed by Holmes [11] and Bapat et al. [1]; Tufillaro and Albano [16] and Wiesenfeld and Tufillaro [17] have performed experiments with this system.

Schaal and Atkeson [15] have studied almost the exact same system in the context of robotic juggling. Bühler and Koditschek [5] demonstrated a system that juggles a puck on an inclined plane using a bat.

In the area of impulsive manipulation, Higuchi [9] demonstrated linear micropositioning using an electromagnetic coil to deliver an impulsive force. More recently, Yamagata and Higuchi [18] have developed a piezoelectric device to deliver impulsive forces to do micropositioning for precision optical components.

Mirtich and Canny [14] introduced the idea of impulsebased dynamic simulation, in which all contact between objects is modeled as a series of microcollisions.

The limiting cases of impulsive manipulation developed in this paper build upon the results of Huang et al. [12] which presented an analytical formulation and solution of the mechanics of tapping a rotationally symmetric object. These limiting cases examine the mechanics as the impulse delivered by a tap approaches zero. Many of the results of this paper are covered in greater detail in Huang and Mason [13]. The present work also makes use of the eigendirections of Goyal et al. [8] in their analysis of the dynamics of sliding objects.

\section{Vibratory Manipulation in 1D}

We consider in one dimension the problem of determining the object behavior when we deliver a sequence of impacts. We note that if the object is kept in continuous motion (continuous tapping) then it is subject to a constant (Coulomb) frictional force. Suppose the striker moves with some periodic vibration superimposed on a constant velocity. When viewed in a frame moving at that constant velocity (see Figure 1), this system is equivalent to a ball bouncing on a vibrating table - between impacts, the object is subject to a constant acceleration produced by friction whereas the ball is subject to acceleration produced by gravity. This system has been studied in the context of chaotic systems and in the context of robotic juggling.

We can apply the results of these analyses to vibratory manipulation in order to relate parameters of the striker's

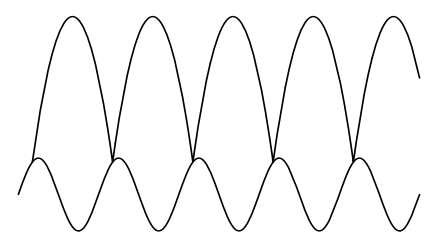

Figure 1: An illustration of regular periodic bouncing. The table moves with a sinusoidal vibration, and the object follows a parabolic trajectory while in flight. This same illustration applies to continuously tapping an object using a striker with a sinusoidal vibration superimposed on a constant velocity (when viewed in the frame of that constant velocity).

motion to possible object behaviors. In this section, we describe some of the main results.

\subsection{The Bouncing Ball Problem}

The problem of a ball bouncing on a sinusoidally vibrating table has been studied in some detail by Holmes [11] and Bapat et al. [1]. This system can exhibit bounded chaotic behavior, though we are primarily interested in regular periodic behaviors. One key result from Holmes is the relation between striker frequency and amplitude for stable periodic bouncing.

We assume the motion of the table is $X(t)=A \sin \omega t$. Holmes formed the return map which maps the velocity of the ball (just before an impact) and the phase of the impact (relative to the table oscillation) from one impact to the next and showed that this return map has a number of fixed points which correspond to different modes of periodic bouncing. Stability analysis of these fixed points showed that in order to maintain stable periodic juggling, the amplitude of the table vibration must satisfy:

$$
\frac{n \pi \mu g}{\omega^{2}} \frac{(1-e)}{(1+e)}<A<\frac{\mu g}{\omega^{2}} \sqrt{n^{2} \pi^{2} \frac{(1-e)^{2}}{(1+e)^{2}}+1}
$$

where the object motion has period one and strikes the table every $n$ table cycles; $e$ is the coefficient of restitution between the object and the striker, and $\mu$ the coefficient of friction between the object and the surface. This relationship is shown graphically in Figure 2.

\subsection{Robotic Juggling}

Schaal and Atkeson [15] have implemented paddle jugging using a "trampoline-like" racket and a ping-pong ball. They observed that negative acceleration of the paddle trajectory at impact seems to provide stability. They calculated the basin of attraction for periodic juggling to be 0.257 the area of the viable space of initial conditions. However, for a number of reasons (including mechanical variability and air resistance) the basin of attraction was significantly larger. In fact, they were unable to avoid getting a periodic (mostly period one) juggling pattern.

Bühler and Koditschek [5] demonstrated a system that juggles a puck on an inclined plane using a bat. In order to achieve stable juggling, they developed a "mirror" control law which servos the bat to nonlinearly reflect the position 


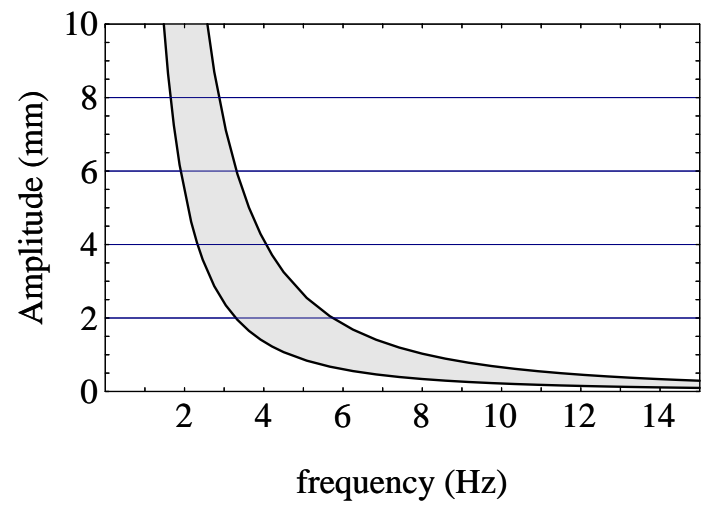

Figure 2: The relationship between the frequency and amplitude for a sinusoidally vibrating striker in order to achieve period one, $n=1$ "bouncing" for vibratory manipulation. Here we have assumed $\epsilon=0.8$ and $\mu=0.25$. Increasing the amplitude beyond the range shown here will result in period doubling and will eventually lead to chaotic behavior. Using an amplitude below this range will likely result in the object coming to rest against the striker and being pushed along.

of the puck. Using this sort of simple control law for vibratory manipulation is appealing but sensing the object would be difficult for high frequency, low amplitude motions.

\section{The Limiting Cases of Impulsive Manipulation}

Vibratory manipulation in two dimensions is much more complicated than in one dimension. There is strong coupling between translational and rotational velocities, and the frictional load on the object is generally a function of the object velocities and the object orientation. It will require greater understanding of the dynamics of a sliding object in order to understand two dimensional vibratory manipulation in general. We have started by formulating the limiting cases of impulsive manipulation for rotationally symmetric objects.

Suppose we manipulate an object by delivering a series of identical taps at some frequency. We take the limiting case to be the limiting behavior of the object as we let the number of taps approach infinity and the impulse of each tap approach zero. Here, we will focus on regular period one motions, i.e. the object will repeatedly follow the same path in state space after each impact. Therefore, we can focus on the resultant object motion from a single tap as the impulse delivered by that tap approaches zero.

In this section, we develop the limiting cases for intermittent tapping and continuous tapping. First, however, we cover a few preliminaries for sliding rotationally symmetric objects in two dimensions.

\subsection{Preliminaries for 2D}

\subsubsection{The Physics of a Sliding Rotating Object}

Rotationally symmetric objects are, for our purposes, objects whose pressure distribution is a function of radius only. These objects have the special property that they slide in a straight line. (Thus we need only consider a two dimensional state space.) As discussed in Huang et al. [12], the net force and torque on a rotationally symmetric object are functions of only the ratio $\frac{\omega}{v}$. The equations of motion are:

$$
\begin{aligned}
& -F\left(\frac{\omega}{v}\right)=m \dot{v} \\
& -T\left(\frac{\omega}{v}\right)=I \dot{\omega}
\end{aligned}
$$

where $F$ and $T$ are the force and torque loads on the object. The initial velocities are $v_{0}$ and $\omega_{0}$.

During the motion, the object will translate a distance $x_{f}$ and rotate an angle $\theta_{f}$

$$
\begin{aligned}
& x_{f}=\int_{0}^{t_{f}} v d t \\
& \theta_{f}=\int_{0}^{t_{f}} \omega d t
\end{aligned}
$$

where $t_{f}$ is the time that the object slides.

\subsubsection{Trajectory Scaling}

Through straightforward use of the chain rule, we can show that if $v(t)$ and $\omega(t)$ are solutions to the equations of motion, then so are $k v\left(\frac{t}{k}\right)$ and $k \omega\left(\frac{t}{k}\right)$. If the first pair of trajectories cover a distance $x_{f}$ and an angle $\theta_{f}$, then the second cover a distance $k^{2} x_{f}$ and an angle $k^{2} \theta_{f}$.

\subsubsection{Centers of Rotation (CORs)}

We can describe any single planar motion of a rigid object by a center of rotation (COR). The COR is the point about which the net movement of the object appears to be a pure rotation. Pure translation corresponds to a COR at infinity. Given the displacement $\vec{x}$ and rotation $\theta$, the COR is located at $\frac{\hat{z} \times \vec{x}}{\theta}$. We can also use CORs to describe the instantaneous motion of an object; given the linear velocity $\vec{v}$ and rotational velocity $\omega$, the COR is located at $\frac{\hat{z} \times \vec{v}}{\omega}$.

We can characterize a mode of manipulation by the locus of CORs which it can effect upon an object. This enables us to compare different modes of manipulation, and it also suffices as the information required in order to create plans for that mode of manipulation.

In this paper, we construct the locus of CORs with respect to the initial translational velocity of the object and assume that rotations are positive. Negative rotations result in a different locus of CORs which is simply the mirror image with respect to the initial velocity.

\subsection{The Limiting Case for Intermittent Tapping}

For intermittent tapping, we deliver a sequence of identical impacts, letting the object come to rest before the next impact. Here, the object repeatedly follows a path in state space starting at the initial velocities and ending at the origin (zero velocities). We take the limiting case to be the 


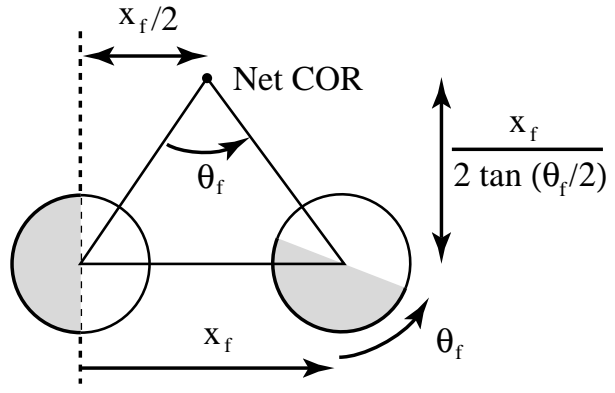

Figure 3: Construction for finding the net COR for a tap where the object moves a distance $x_{f}$ and turns an angle $\theta_{f}$.

resultant behavior as the impulse approaches zero but the ratio of the initial velocities remains the same.

We first determine the net COR for a motion in the limit and then find the locus of CORs possible for this limiting case.

\subsubsection{Net COR in the Limit}

Given the distance traveled and the angle turned, we can determine the net COR for the motion with a geometric construction as illustrated in Figure 3. The location of the net COR is:

$$
\frac{\overrightarrow{x_{f}}}{2}+\frac{\hat{z} \times \overrightarrow{x_{f}}}{2 \tan \frac{\theta_{f}}{2}}
$$

Since we let the impulse of a tap approach zero by scaling the initial velocities, we can apply the trajectory scaling of Section 4.1 .2 (scaling the velocities by $k$ ). In the limit, the COR is then

$$
\lim _{k \rightarrow 0} \frac{k^{2} \overrightarrow{x_{f}}}{2}+\frac{\hat{z} \times k^{2} \overrightarrow{x_{f}}}{2 \tan \frac{k^{2} \theta_{f}}{2}}=\lim _{k \rightarrow 0} \frac{\hat{z} \times k^{2} \overrightarrow{x_{f}}}{2 \tan \frac{k^{2} \theta_{f}}{2}}
$$

Applying l'Hôpital's rule (differentiating both numerator and denominator with respect to $k$ ), we get

$$
\lim _{k \rightarrow 0} \frac{\hat{z} \times \overrightarrow{x_{f}}}{\theta_{f} \sec ^{2} \frac{k^{2} \theta_{f}}{2}}=\frac{\hat{z} \times \overrightarrow{x_{f}}}{\theta_{f}}
$$

Thus, as the impulse delivered by a tap approaches zero, the distance from the center of mass (COM) to the net COR approaches $\frac{x_{f}}{\theta_{f}}$.

\subsubsection{Locus of CORs}

In order to characterize this limiting case, we construct the locus of CORs. This represents all possible motions of the object for a given initial velocity direction.

The trajectory scaling of Section 4.1.2 shows that each value of $\frac{\omega_{n}}{v_{0}}$ corresponds to a single value of $\frac{x_{f}}{\theta_{f}}$, no matter what the magnitude of the initial velocities or the resulting displacements. In other words $\frac{x_{f}}{\theta_{f}}$ is a function of $\frac{w_{0}}{v_{0}}$. Huang and Mason [13] further showed that $\frac{x_{f}}{\theta_{f}}$ is a continuous monotonic decreasing function of $\frac{\omega_{n}}{v_{0}}$. Therefore the minimum and maximum values of $\frac{\omega_{0}}{v_{0}}$ that we can achieve via an impact correspond to the maximum and minimum values of $\frac{x_{f}}{\theta_{f}}$ for the resulting displacements. These values of $\frac{x_{f}}{\theta_{f}}$ in turn determine the closest and furthest CORs possible for this limiting case.

Huang et al. [12] formulated the impact cone which represents the set of velocities that can be achieved by striking an object at rest. We can restate this cone in terms of the ratios of initial velocities possible

$$
\frac{\omega_{0}}{v_{0}} \leq \frac{\mu_{r} R M}{I \sqrt{1+\mu_{r}^{2}}}
$$

The minimum value of $\frac{\omega_{0}}{v_{0}}$ is 0 , corresponding to pure translational velocity (which results in a pure translation of the object: $\frac{x_{f}}{\theta_{f}}=\infty$ ); the maximum value is given by the equality condition of Equation 9.

Let $c_{\min }=\frac{x_{f}}{\theta_{f}}$ for the maximum value of $\frac{\omega_{n}}{v_{n}}$ that satisfies Equation 9. The locus of CORs possible for the limiting case of intermittent tapping is then

$$
\left\{c \hat{z} \times \hat{v}_{0} \mid c \in\left[c_{\min }, \infty\right]\right\}
$$

\subsection{The Limiting Case of Continuous Tapping in 2D}

For continuous tapping, we deliver a sequence of regularly spaced impacts, always striking the object before it comes to rest. Here, the object follows a path in state space starting at its initial velocities and continues until the object is tapped again, "kicking" the state back to the original initial velocities. We take the limiting case to be the resulting behavior of the object as the amount of time before the next impact approaches zero.

We take an analogous approach (to that for the limiting case of intermittent tapping) to determine the location of the net COR in the limit. We start with the same expression (Equation 6) for the net COR given the displacement and rotation of the object:

$$
\frac{\overrightarrow{x_{f}}}{2}+\frac{\hat{z} \times \overrightarrow{x_{f}}}{2 \tan \frac{\theta_{f}}{2}}
$$

As we decrease the time that the object slides $\left(t_{f}\right), \overrightarrow{x_{f}}$ and $\theta_{f}$ both approach zero:

$$
\lim _{t_{f} \rightarrow 0} \frac{\overrightarrow{x_{f}}}{2}+\frac{\hat{z} \times \overrightarrow{x_{f}}}{2 \tan \frac{\theta_{f}}{2}}=\lim _{t_{f} \rightarrow 0} \frac{\hat{z} \times \overrightarrow{x_{f}}}{2 \tan \frac{\theta_{f}}{2}}
$$

Assume for the moment that this limit exists. Applying l'Hôpital's rule (differentiating numerator and denominator with respect to $\left.t_{f}\right)$, we get

$$
\begin{aligned}
\lim _{t_{f} \rightarrow 0} \frac{\hat{z} \times \frac{d}{d t_{f}} \overrightarrow{x_{f}}}{\sec ^{2} \theta_{f} \frac{d}{d t_{f}} \theta_{f}}=\lim _{t_{f} \rightarrow 0} \frac{\hat{z} \times \frac{d}{d t_{f}} \int_{0}^{t_{f}} \vec{v} d t}{\frac{d}{d t_{f}} \int_{0}^{t_{f}} \omega d t}= \\
\lim _{t_{f} \rightarrow 0} \frac{\hat{z} \times \vec{v}\left(t_{f}\right)}{\omega\left(t_{f}\right)}=\frac{\hat{z} \times \overrightarrow{v_{0}}}{\omega_{0}}
\end{aligned}
$$

Thus, the COR in the limit is simply the initial (instantaneous) COR.

The remainder of this subsection examines the conditions under which this limit exists and then finds the locus of CORs possible under this limiting case. 


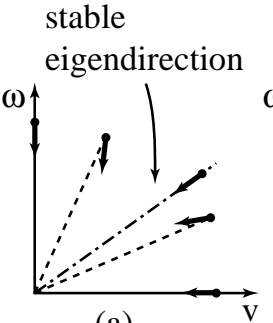

(a) negative impact cone

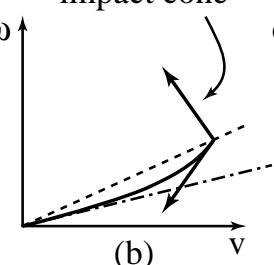

(b) negative

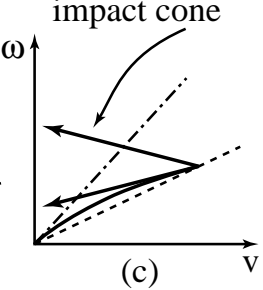

Figure 4: Illustrations of the state space for a rotating sliding rotationally symmetric object; the stable eigendirection is shown as the dashed-dotted line. Figure (a) shows a typical vector field, which is a function of only $\frac{\omega}{v}$. Figure (b) illustrates the case where the initial slope of the path in state space aligns with the negative impact cone above the stable eigendirection. In this case, the trajectory curves in to the cone. Figure(c) illustrates the case where this alignment occurs below the eigendirection; in this case, the trajectory curves away from the cone.

\begin{tabular}{|l|r|r||r|r|}
\cline { 2 - 5 } \multicolumn{1}{c|}{} & \multicolumn{2}{c||}{ Disc } & \multicolumn{2}{c|}{ Ring } \\
\cline { 2 - 5 } & $\frac{\omega}{v}$ & COR & $\frac{\omega}{v}$ & COR \\
\hline \hline Intermittent LC & & 0.080 & & 0.208 \\
\hline Continuous LC & 16.395 & 0.061 & 8.951 & 0.112 \\
\hline Impact Cone & 9.704 & 0.103 & 4.851 & 0.206 \\
\hline Eigendirection & 30.798 & 0.032 & 20.000 & 0.050 \\
\hline Radius & & 0.050 & & 0.050 \\
\hline
\end{tabular}

Table 1: Motion constraints for a disc and a ring for both limiting cases (LCs). The distance to the COR given for the impact cone, limiting cases, and pushing is the distance to the closest COR. The distance to the COR corresponding to the stable eigendirection and the radius of the object are given for comparison. Quasistatic pushing has the same motion constraints as the limiting case of continuous tapping. These results were numerically generated, and assume that $\mu=\mu_{r}=0.25$.

\subsubsection{Impact Constraint for Moving Objects}

For the limiting case of intermittent tapping, we reformulated the impact cone (the cone in state space that represents the velocities that can be achieved by tapping an object at rest) in terms of the achievable ratios of $\frac{\omega_{0}}{v_{0}}$. For a given point in the impact cone, we can compute the striker velocity and contact point required to achieve that change in velocity. Not surprisingly, so long as we recreate the same relative velocities between the striker and the object, we can achieve the same change in velocity. This implies that we can restate Equation 9 in terms of change in velocities

$$
\frac{|\Delta \omega|}{\Delta v} \leq \frac{\mu_{r} R M}{I \sqrt{1+\mu_{r}^{2}}}
$$

The result is that we can move the impact cone to any point in state space.

We will primarily make use of the negative impact cone, which we define as the set of states from which we can reach the current state with a single impact. Since the impact cone is invariant to its position in state space, we form the negative impact cone by simply reflecting the impact cone through the current state.

\subsubsection{Existence of the Limit \& Locus of CORs}

The limit taken in Equations 12 and 13 exists if and only if the path of the object in state space passes through the negative impact cone (at the initial velocities) for a nonzero period of time. In order to determine which initial conditions satisfy this property, we must examine properties of paths in state space. The eigendirections of Goyal et al. [8] serve to guide our analysis.

There are a number of properties of paths in state space for a sliding rotationally symmetric object. We state them here in terms of the vector field $(\dot{v}, \dot{\omega})=(-F / M,-T / I)$ over the state space.

- as $\frac{\omega}{v}$ increases, the orientation of $(\dot{v}, \dot{\omega})$ monotonically increases from $\pi$ to $\frac{3}{2} \pi$.

- $(\dot{v}, \dot{\omega})$ points towards the origin only at values of $\frac{\omega}{v}$ corresponding to eigendirections; most objects have a single stable eigendirection that is neither pure translation nor rotation.

- at all points below the stable eigendirection, $(\dot{v}, \dot{\omega})$ points above the origin (and vice versa), drawing the system towards the stable eigendirection.

These properties are illustrated in Figure 4a.

If we place the negative impact cone on the line corresponding to $\frac{\omega}{v}=0$ (a COR at infinity), the path in state space will lie entirely inside the cone. As we increase the value of $\frac{\omega}{v}$, the vector $(\dot{v}, \dot{\omega})$ will rotate counterclockwise. The path of the object in state space will continue to pass through the cone until this vector aligns with the bottom edge of the cone. At that point, we have

$$
\frac{F\left(\frac{\omega}{v}\right)}{T\left(\frac{\omega}{v}\right)}=\frac{R}{\sqrt{1+\frac{1}{\mu_{r}^{2}}}}
$$

Let $1 / c_{\min }$ be the value of $\frac{\omega_{0}}{v_{0}}$ which satisfies this equation.

If this alignment occurs above the stable eigendirection then the path will curve into the cone (see Figure 4b), so the set of possible CORs is

$$
\left\{c \hat{z} \times \hat{v}_{0} \mid c \in\left[c_{\min }, \infty\right]\right\}
$$

If this alignment occurs below the stable eigendirection then the path will curve away from the cone (see Figure 4c), so the set of possible CORs is

$$
\left\{c \hat{z} \times \hat{v}_{0} \mid c \in\left(c_{\min }, \infty\right]\right\}
$$

Interestingly, the set of possible CORs for pushing an object is identical to Equation 16. Pushing can achieve any COR so long as $\frac{\omega}{v}$ corresponds to a force/torque load due to an applied force in the friction cone (in "force space") at the contact point; the limiting case of continuous tapping can achieve any COR so long as $\frac{\omega_{0}}{v_{0}}$ results in a force/torque within the negative impact cone, which is simply a transformation of the friction cone (in impulse space) at the contact point. The mapping between force/torque due to friction and the object velocities is the same for both modes of manipulation. 


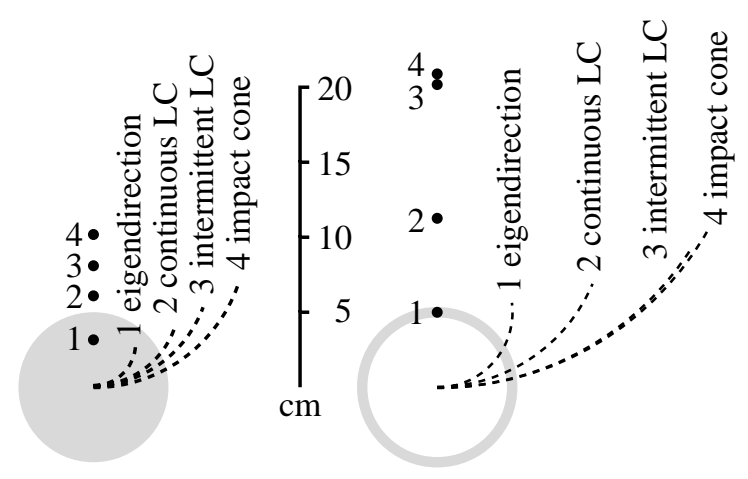

Figure 5: Graphic illustration of the motion constraints on a disc and a ring due to tapping. Points $2-4$ are the closest COR achievable via the respective mode of manipulation. The full set of CORs include all the points vertically above, going out to infinity. Point 1 represents the stable eigendirection; the dotted lines show the path the object would take if it continued to move about that COR.

\section{Examples for the Limiting Cases}

Table 1 shows the results of analyzing the possible motions of a uniform disc and a ring under the two limiting cases. These results are graphically illustrated in Figure 5. Note that the "alignment" for the limiting case of continuous tapping occurs below the stable eigendirection (i.e. the value of $\frac{\omega}{v}$ (slope in state space) for the limiting case is less than that for the eigendirection).

\section{Summary}

In this paper, we developed the idea of vibratory manipulation in the context of objects sliding on a planar surface. We found two distinct modes of vibratory manipulation: intermittent tapping, where the object comes to rest between impacts, and continuous tapping, where the object is always tapped again before it comes to rest.

We first examined one dimensional continuous tapping; this mode of manipulation is closely related to bouncing a ball on a vibrating table. We applied work on the bouncing ball problem to determine the relationship between striker frequency and amplitude in order to achieve regular periodic bouncing under continuous tapping. Research in robotic juggling suggests that the basin of attraction for stable periodic bouncing is larger than theory indicates.

The two dimensional case of vibratory manipulation is more complex. Vibratory manipulation (which concerns object behavior from repeated impacts) is an approximation to the limiting cases of impulsive manipulation (which addresses the mechanics of a single impact). We developed the limiting cases for rotationally symmetric objects and found that the possible motions for the limiting case of continuous tapping are (nearly) identical to those for quasistatic pushing. Finally, we applied our analysis to determine the possible motions for a disc and a ring under both limiting cases.

\section{References}

[1] C. N. Bapat, S. Sankar, and N. Popplewell. Repeated impacts on a sinusoidally vibrating table reappraised. Journal of Sound and Vibration, 108(1):99-115, 1986.

[2] K. Böhringer, V. Bhatt, and K. Goldberg. Sensorless manipulation using transverse vibrations of a plate. In IEEE International Conference on Robotics and Automation, volume 2, pages 1989-96, 1995.

[3] G. Boothroyd. Assembly Automation and Product Design. Marcel Dekker, 1992.

[4] G. Boothroyd, C. Poli, and L. E. Murch. Automatic Assembly. Marcel Dekker, 1982.

[5] M. Bühler and D. E. Koditschek. From stable to chaotic juggling: Theory, simulation, and experiments. In IEEE International Conference on Robotics and Automation, pages 1976-1981, Cincinnati, OH, 1990.

[6] M. Caine. The design of shape interactions using motion constraints. In IEEE International Conference on Robotics and Automation, 1994.

[7] A. Christiansen, A. Edwards, and C. Coello. Automated design of part feeders using a genetic algorithm. In IEEE International Conference on Robotics and Automation, volume 1, pages 846-51, 1996.

[8] S. Goyal, A. Ruina, and J. Papadopoulos. Planar sliding with dry friction. Part 1. Limit surface and moment function. Wear, 143:307-330, 1991.

[9] T. Higuchi. Application of electromagnetic impulsive force to precise positioning tools in robot systems. In International Symposium on Robotics Research, pages 281-285. Cambridge, Mass: MIT Press, 1985.

[10] H. Hitakawa. Advanced parts orientation system has wide application. Assembly Automation, 8(3):147-150, 1988.

[11] P. J. Holmes. The dynamics of repeated impacts with a sinusoidally vibrating table. Journal of Sound and Vibration, 84(2):173-189, 1982.

[12] W. Huang, E. P. Krotkov, and M. T. Mason. Impulsive manipulation. In IEEE International Conference on Robotics and Automation, 1995.

[13] W. H. Huang and M. T. Mason. Limiting cases of impulsive manipulation. Technical Report CMU-RI-TR-96-24, The Robotics Institute, Carnegie Mellon University, September 1996.

[14] B. Mirtich and J. Canny. Impulse-based dynamic simulation. In K. Goldberg, D. Halperin, J.-C. Latombe, and R. Wilson, editors, Algorithmic Foundations of Robotics, pages 407-418. A. K. Peters, Boston, MA, 1995.

[15] S. Schaal and C. G. Atkeson. Open loop stable control strategies for robot juggling. In IEEE International Conference on Robotics and Automation, pages 3:913-918, Atlanta, GA, 1993.

[16] N. B. Tufillaro and A. M. Albano. Chaotic dynamics of a bouncing ball. American Journal of Physics, 54(10):939944, October 1986.

[17] K. Wiesenfeld and N. B. Tufillaro. Suppression of period doubling in the dynamics of a bouncing ball. Physica D, 26(1-3):321-335, May-June 1987.

[18] Y. Yamagata and T. Higuchi. A micropositioning device for precision automatic assembly using impact force of piezoelectric elements. In IEEE International Conference on Robotics and Automation, 1995. 